\title{
Romain Rolland dans le contexte suisse de la Grande Guerre
}

Guillaume Juin

\section{(2) OpenEdition}

1 Journals

Édition électronique

URL : http://journals.openedition.org/edl/342

DOI : $10.4000 /$ edl.342

ISSN : 2296-5084

Éditeur

Université de Lausanne

\section{Édition imprimée}

Date de publication : 15 septembre 2012

Pagination : 75-104

ISBN : 978-2-940331-28-4

ISSN : 0014-2026

\section{Référence électronique}

Guillaume Juin, "Romain Rolland dans le contexte suisse de la Grande Guerre », Études de lettres [En ligne], 3 | 2012, mis en ligne le 15 septembre 2015, consulté le 18 décembre 2020. URL : http:// journals.openedition.org/edl/342; DOI : https://doi.org/10.4000/edl.342 


\section{ROMAIN ROLLAND DANS LE CONTEXTE SUISSE DE LA GRANDE GUERRE}

Romain Rolland ne choisit pas la Suisse en 1914. Il s'y trouvait lorsque la guerre éclata et il décida d'y demeurer afin de mener un combat humaniste et pacifiste au nom de valeurs alors menacées. Il fut rejoint en Suisse par d'autres dissidents français en 1915, tandis qu'il quittait le combat éditorial qu'il avait initié. Il devint dès lors une figure morale pour beaucoup, bien malgré lui.

\section{Le choix de Romain Rolland en 1914: la Suisse, au chevet d'une Europe déchirée}

\subsection{La Suisse pendant la guerre ${ }^{1}$}

La neutralité de la Suisse est un statut permanent entré dans l'ordre public européen en 1815, lui conférant une abstention volontaire dans la participation à la guerre des autres. Le Conseil fédéral s'engage donc en 1914 à maintenir et défendre cette neutralité. Pour ce faire, le gouvernement ordonne la mobilisation générale pour le $1^{\mathrm{er}}$ août 1914 . Dans un climat de patriotisme et une ambiance parfois agitée, 250000 hommes composent l'armée de milice: chiffre considérable en comparaison des mobilisations antérieures, mais chiffre atténué par des moyens matériels insuffisants.

En réalité, la Suisse se concentre surtout à protéger son indépendance et sa frontière. Frontière exposée du fait de la situation du pays au cœur

I. Titre d'un article de J.-C. Favez dans S. Audoin-Rouzeau, J.-J. Becker (éds), Encyclopédie de la Grande Guerre 1914-1918, p. 867. 
de l'Europe et dont la frontière touche deux belligérants qui s'opposent, l'Allemagne et la France. La Suisse modèle ses stratégies en fonction des belligérants. Et tant qu'elle ne serait pas attaquée, elle resterait fidèle à sa neutralité.

Un premier problème allait survenir: celui de différends sur le territoire entre Suisse romande et Suisse alémanique. En raison d'une part de leur position géographique à proximité de la France pour l'une et de l'Allemagne pour l'autre et, d'autre part, d'une sorte de communautarisme linguistique, ces différends sont entretenus par la presse. Ainsi, la violation de la neutralité belge dès le 4 août 1914 par l'Allemagne soulève l'indignation en terre romande, alors que la Suisse alémanique cherche une excuse. Romain Rolland commentera ces différends entre les deux Suisse:

Je vois autour de moi frémir la Suisse amie. Son cœur est partagé entre des sympathies de races différentes; elle gémit de ne pouvoir librement choisir entre elles, ni même les exprimer ${ }^{2}$.

Ce cœur partagé sera même divisé à la fin de 1915 par l'affaire dite "des colonels». Le chef du service de renseignement de l'armée et son premier collaborateur ont transmis aux Puissances centrales des informations confidentielles, ce qui viole la neutralité en faveur de l'Allemagne. Lopinion publique romande obligera le gouvernement à déférer les deux hommes à la justice. Cette affaire aura des conséquences dans les relations entre les communautés linguistiques, en témoigne plusieurs actes de violence commis en Suisse romande entre 1915 et 1917 contre la présence germanique.

De même, au printemps 1917, l'homme fort du gouvernement et leader socialiste Arthur Hoffmann se laisse entraîner - et entraîne avec lui Robert Grimm, jeune et actif député leader de l'aile gauche du Parti socialiste suisse - dans des contacts avec les Allemands. Le scandale est découvert, il est énorme. Hoffmann est remplacé par Gustav Ador, libéral et francophile, reconnu sur la scène internationale grâce à sa présidence du Comité International de la Croix-Rouge (CICR).

Enfin, la grève générale que connaît la Suisse à partir du 12 novembre 1918 illustre les difficultés économiques et sociales que le pays tente de contenir. De la survie économique du pays jusqu'au rationnement trop

2. R. Rolland, Au-dessus de la mêlée, p. 61. 
tardif en passant par la hausse des prix, le monde ouvrier, les syndicats et le parti socialiste manifestent de plus en plus une volonté de rupture.

Cette rupture est consommée en novembre 1918 à l'appel du comité d'Olten ${ }^{3}$ et se matérialise par une grève générale, événement essentiel de l'histoire helvétique depuis la création de l'Etat fédéral moderne en 1848. On le voit, la Suisse ne traversa pas la guerre sans problème ni difficulté. Mais elle mena une politique de neutralité active dont le symbole fort reste l'œuvre du CICR et de son Agence internationale des prisonniers de guerre basée à Genève. Une ville qui connut elle aussi son lot de réactions face à la guerre, une ville dont la vocation pacifiste sera de nouveau affirmée à partir de 1914.

\subsection{Genève face à la guerre}

\section{Les réactions}

L'année 1914 coïncide avec le centenaire de l'agrégation de Genève à la Confédération suisse. C'est aussi l'année du déclenchement du premier conflit mondial. L'atmosphère genevoise du mois de juin et du début du mois de juillet est une atmosphère de joie et d'enthousiasme patriotique agrémentée de fêtes, discours et célébrations. C'est dire si l'annonce de «la mobilisation complète en Russie et l'état de guerre proclamée en Allemagne» ${ }^{4}$ provoque un sentiment de tourmente et de folie à Genève. Les journaux offrent alors une tragique alternance de nouvelles. La mobilisation à Genève se fait dans la journée du $1^{\text {er }}$ août alors que la population civile suit avec stupeur les premiers évènements de la guerre. L'invasion de la Belgique au cours de ce même mois, suivie de la destruction de Louvain, sont très mal ressenties à Genève. Elles sont vues comme une odieuse violation du droit des gens. Cette attaque injustifiable à l'égard d'un Etat pacifique et neutre - la Belgique - souleva dans la ville une véhémente indignation et une intense émotion.

3. Ainsi avait été baptisé le comité national de lutte regroupant partis et syndicats, Olten étant la ville du canton de Soleure qui les avait accueillis.

4. C'est notamment par ce télégramme du Conseil fédéral affiché à la gare de Vevey le 31 juillet 1914 que Romain Rolland apprend la nouvelle du commencement du "grand égorgement». 
La problématique de la neutralité

La question de la neutralité de la Suisse resurgit alors dans le débat genevois. Deux exemples montrent que cette question reste profondément liée à l'identité suisse: le premier est la réponse du professeur Alfred Martin dans Le Journal de Genève du 5 septembre 1914 à un article d'un professeur de Berne qui préconisait la "neutralité morale»:

La neutralité morale n'est pas autre chose que la négation de la morale. Il est contraire à l'essence de la morale d'être neutre, c'est-à-dire indifférent au bien et au $\mathrm{mal}^{5}$.

Le second exemple est le discours d'Henri Fazy, président du Conseil d'Etat de Genève et doyen d'âge du Conseil national, lors de l'ouverture de la session des Chambres fédérales le 7 décembre 1914:

Neutralité n'est pas synonyme d'indifférence. Oui, Messieurs, tout en restant neutres, nous conservons le droit d'apprécier les évènements contemporains, et de les apprécier en nous plaçant sur le territoire de la Justice, qui est éternelle et qui est la grande loi de l'histoire ... ${ }^{6}$

Ces deux exemples montrent que les hommes politiques, les écrivains, les intellectuels se mobilisèrent pour donner une expression réfléchie et mesurée aux sentiments citoyens. Les deux personnalités que furent Carl Spitteler et Paul Seippel s'employèrent aussi en 1914 par des discours, des articles ou des brochures à témoigner de leur prise de positions.

Le premier fut une des grandes amitiés de Rolland en Suisse allemande, poète suisse d'expression allemande qui reçut le Prix Nobel de littérature en 1919. Le second fut lié amicalement à Rolland pendant près de vingt ans. Une amitié qui fut, parmi ses amitiés suisses, l'une des plus pures et des plus fécondes, malgré l'adhésion passive de Seippel à la fatalité.

Paul Seippel tenta par exemple d'opérer un rapprochement entre la Suisse romande et la Suisse alémanique. Voici comment il décrit la situation le 28 août 1914 :

5. Cité dans "Genève pendant la Première guerre mondiale", in Histoire de Genève de 1798 à 1931, p. 414.

6. Ibid. 
Je suis triste à pleurer. Et tout Genève a l'air d'une ville en deuil. C'est maintenant que la France est si menacée qu'on sent à quel point on l'aime ${ }^{7}$.

Né à Lausanne en 1858, Paul Seippel fut professeur de littérature française à l'Ecole polytechnique fédérale de Zurich, critique littéraire puis directeur du Journal de Genève au début de la guerre. Il fut notamment le premier critique à parler de Jean-Christophe, roman-fleuve de Romain Rolland, au public européen, dans Le Journal de Genève en 1905.

\section{L'œuvre de la Croix-Rouge}

Genève reste le symbole du mouvement humanitaire par le rôle joué par le CICR et par la mise en place de l'Agence internationale des prisonniers de guerre le 21 août 1914. Cette vocation humanitaire date de 1863, avec la fondation de la Croix-Rouge à l'instigation d'Henri Dunant.

C'est véritablement avec la Première Guerre mondiale que le CICR acquiert sa légitimité, du fait de son action plus visible et plus directe. Présidé par Gustave Ador ${ }^{8}$, il met en place en 1914 l'Agence internationale des prisonniers de guerre pour servir d'intermédiaire entre les prisonniers de toutes les nations et les familles.

Genève apparait donc en 1914, comme elle l'était déjà apparue avant, comme une de ces villes où agir moralement et humainement semble lié à l'identité urbaine de la cité. Neutre et idéaliste, Genève réagit à la guerre comme elle avait toujours réagi face aux évènements historiques européens:

On pourrait même affirmer qu'elle pourrait prétendre à une profondeur historique qu'il serait difficile à d'autres lieux de référence ou d'étape de la longue marche de l'Europe vers son unité [...] La vocation "européenne" de Genève s'enracine dans les mouvements démocratiques et pacifistes qui émergent dans les premières décennies du XIX ${ }^{\mathrm{e}}$ siècle?

7. R. Rolland, Journal des années de guerre (cité par la suite $J A G$ ), p. 43.

8. Né en 1845 à Genève, il effectue d'abord une brillante carrière juridique et politique, faisant partie de nombreuses commissions et missions fédérales.

9. A. Fleury, "Genève», p. 89. 
C'est dans ce contexte genevois, conscient que la ville suisse pourra l'aider et le servir, que Romain Rolland décide de demeurer en Suisse pendant toute la période du conflit. Mais il faut noter aussi une relative réserve de Romain Rolland, à certains moments de son séjour genevois, à l'égard d'une ville qui baigne parfois dans un véritable climat de ferveur francophile.

\subsection{Le choix genevois de Romain Rolland en 1914}

\section{Romain Rolland et la Suisse}

Romain Rolland ne découvre pas la Suisse subitement en 1914. Il avait l'habitude d'y passer ses vacances voire de plus longs moments depuis sa découverte du pays en septembre 1882. Mais Romain Rolland n'est pas non plus devenu de nationalité suisse, comme certains ont pu le croire ${ }^{10}$. Son attachement au pays est profond et fidèle, Romain Rolland s'installant même à la Villa Olga, à Villeneuve, le 30 avril 1922, où il résidera pendant de longues années. Il apprécie ses montagnes et ses paysages, son air vivifiant, et y vient parfois soigner sa santé chancelante. Que ce soit Zurich, Bâle, Genève, Lausanne, Vevey ou Villeneuve, Romain Rolland trouvait en Suisse le repos et le calme lui permettant d'écrire. Jean-Christophe est par exemple écrit pour l'essentiel pendant les vacances d'été passées en Suisse. Il se permet ces voyages en raison du confort que lui assure sa position d'intellectuel: « ... ayant par [ses] écrits conquis l'indépendance matérielle suffisante pour vivre sans luxe, mais à l'écart (le plus grand des luxes)» ${ }^{11}$, Rolland aime cette Suisse, apprécie ce carrefour des cultures:

Les villes de Suisse sont les intermédiaires entre la pensée française et la pensée germanique. En très peu de jours, on renouvelle son fonds intellectuel pour plusieurs mois ${ }^{12}$.

Réciproquement, la Suisse aime Rolland. Beaucoup d'intellectuels, écrivains, poètes suisses sont ses amis. Nous en avons déjà évoqué deux,

Io. Henri Massis par exemple: «Romain Rolland n'est pas Suisse, comme on le croit communément. Il est né en France.» cité in H. Guilbeaux, Pour Romain Rolland.

II. Catalogue d'exposition Romain Rolland et la Suisse, p. 13.

I2. Ibid., p. 9. 
Spitteler et Seippel. Ajoutons Edmond Privat, né en 1889, qui fut le propagandiste et le propagateur non seulement de l'œuvre de Romain Rolland, mais aussi de ses idées. Leur formidable correspondance a été publiée sous le beau titre de Bon voisinage, car Edmond Privat s'installa pendant l'entre-deux-guerres à Veytaux, près de Villeneuve. Privat comprit vite le choix de Rolland en 1914:

Privat me parle de l'hostilité que mes articles ont soulevée contre moi à Paris. On n’admet point que je reste à Genève. On me soupçonne. Mais Privat m'approuve, car il pense

$1^{\mathrm{o}}$ que si je revenais, je ne pourrais plus parler;

$2^{\circ}$ que si je parlais, je serai assassiné ${ }^{13}$.

D'autres personnalités suisses liées à l'engagement de Rolland en 1914 comme Gustave Ador ou Frédéric Ferrière sont aussi à mentionner, car ils influencèrent également l'intellectuel français.

L'été 1914

En juin 1914, Romain Rolland est en Suisse pour y passer l'été. Dès le 2, il arrive à Vevey. Victor Hugo disait y apprécier «la propreté, le climat et l'église». Romain Rolland y était en juin 1914, à l'hôtel Mooser, se promenant en ville et observant ses livres en devanture des librairies de Vevey où il était désormais connu. Son mois de juin est fait d'excursions dans la montagne suisse, de retouches à son manuscrit de Colas Breugnon. A la fin du mois, il écrit à sa mère: "J'ai à peu près terminé ma correction du manuscrit de Colas Breugnon. Je suis dans un état de liberté d'esprit. ${ }^{14}$.

Du 2 au 7 juillet, Romain Rolland est à Genève, avec son ami Alphonse de Châteaubriant. Ils suivent les grandes cérémonies nationales liées au centenaire du rattachement de Genève à la Confédération suisse. Coupé de la France géographiquement, mais non intellectuellement, Romain Rolland suit les évènements français comme celui de l'affaire

I3. $J A G$, p. 330 .

I4. Lettre à sa mère, 22 juin 1914. La correspondance avec sa mère est inédite pour les mois qui précèdent la guerre puisque la première lettre de R. Rolland, Je commence à devenir dangereux, est datée du 9 novembre 1914. 
Caillaux. Henriette Caillaux, épouse de Joseph Caillaux ${ }^{15}$, tue de plusieurs coups de revolver Gaston Calmette, directeur du Figaro, qui menait une campagne de presse contre son époux. Le procès qui s'ouvre en juillet se termine par l'acquittement de $\mathrm{M}^{\text {me }}$ Caillaux. Romain Rolland notera à propos de cette affaire:

Lis-tu l'affaire Caillaux? Je la suis attentivement, et toutes mes sympathies sont pour Caillaux et surtout pour $\mathrm{M}^{\text {me }}$ Caillaux. Ces journaux sont hideux. Une bande de chiens lâchés sur une proie ${ }^{16}$.

Romain Rolland est déjà critique vis-à-vis d'une presse dont il sera luimême la victime quelques mois plus tard.

Rolland ne quitte donc pas la France en juillet 1914 pour se réfugier en Suisse. Il ne s'exile pas ni ne fuit son pays. Sa présence en Suisse est une habitude, elle n'est pas surprenante. Et quand se déclenche la guerre, son choix sera celui de Vevey puis celui de Genève, comme si cela semblait naturel de demeurer dans un pays qui avait l'habitude de l'accueillir.

Le choix de Romain Rolland au moment du déclenchement du conflit

Tout le monde n'a pas eu le choix, en août 1914, de partir au front ou non. Louis Gillet, ami fidèle de Rolland, correspondant avec lui depuis $1897^{17}$, lui écrit le 11 août 1914 :

J'ai vécu quinze jours qui en valent la peine, quinze jours que je n'osais plus me promettre du destin. On parlera de nous dans l'histoire. Nous aurons ouvert une ère dans le monde ${ }^{18}$.

Agé alors de 48 ans, Romain Rolland n'est pas mobilisable. De plus, sa santé le dispense de toute obligation militaire. Victime d'un accident de voiture en 1910, l'homme grand et frêle a depuis quelques soucis de santé. Par exemple en 1913, un voyage en Bourgogne le fatigue. Victime

I5. Président du Conseil (1911-1912), membre du parti radical, ministre des Finances en 1914. Il est arrêté en 1917 pour "correspondance avec l'ennemi».

I6. Lettre à sa mère, 27 juillet 1914 .

17. Cité dans Correspondance entre Louis Gillet et Romain Rolland.

I8. Id., p. 20. 
d'oppression, il est pris d'insomnies. La souffrance morale qui sera parfois la sienne aura aussi des conséquences sur sa santé physique.

Son âge et sa santé le dispensent donc des obligations militaires et lui permettent de faire le choix de demeurer en Suisse, malgré un bref retour en France pour récupérer quelques affaires. Mais ce choix n'est pas fixé dès le début des hostilités: "Je vais rentrer prochainement à Paris. [...] Nous souffrons moins que vous; mais nous nous tourmentons bien davantage. ${ }^{19}$.

Cette réponse à Louis Gillet est datée du 28 août, et dès le lendemain Rolland songe à se "mettre à la disposition du consulat, qui est débordé ${ }^{20}$ ». Finalement, il reste à Genève et confiait déjà les 3-4 août:

Je suis accablé. Je voudrais être mort. Il est horrible de vivre au milieu de cette humanité démente, et d'assister, impuissant, à la faillite de la civilisation. Cette guerre européenne est la plus grande catastrophe de l'histoire, depuis des siècles, la ruine de nos espoirs les plus saints en la fraternité humaine ${ }^{21}$.

Puis les 5-7 août:

Je me trouve seul, exclu de cette communion sanglante. Une fois de plus, je me sens, comme dans l'Affaire Dreyfus, isolé du reste des hommes. Et je cherche à comprendre pourquoi je suis ainsi, et d'où me vient ce don funeste de ne pouvoir m'associer à aucun des grands mouvements humains ${ }^{22}$.

Et enfin le 22 août:

Ma souffrance est une somme de souffrances, si compacte et serrée qu'elle ne me laisse plus l'espace de respirer. C'est l'écrasement de ma France, sa ruine définitive. C'est le sort de mes amis, morts peutêtre ou blessés. C'est l'horreur de ces souffrances, la communion déchirante avec ces millions de malheureux ${ }^{23}$.

\footnotetext{
19. Ibid.

20. Cité dans Chère Sofia, t. II, p. 209.

2I. $J A G$, p. 32.

22. Ibid., p. 34.

23. Ibid., p. 38.
} 
Ce mois d'août fut donc celui d'une grande souffrance pour Romain Rolland. Mais ce guide moral dont il parle, c'est bien lui qui le jouera de Suisse. Dès ce même mois de souffrances, Romain Rolland lance son combat pour la justice et la morale, signe que dans l'accablement et la souffrance, il fut capable de puiser la force nécessaire pour réagir.

S'il demeure à Vevey pendant les deux premiers mois du conflit, c'est bien par Genève que l'activité et l'action passent. Il s'y rend le 31 août 1914:

Je vais à Genève, convoqué par Seippel à la première réunion d'une Commission Internationale, pour protester contre la destruction de Louvain ${ }^{24}$. Mais à cette séance n'assistent qu'une quinzaine de notabilités genevoises qui, à la quasi-unanimité, se prononcent «... pour ne rien faire qui puisse les compromettre...» [...] L’attitude épeurée de ces représentants de la grande vieille bourgeoisie de Genève m'indigne; mais je (n’en) puis rien manifester: seul étranger, au milieu de ces Suisses, je n'ai pas le droit d'intervenir dans leurs débats nationaux. Je bous intérieurement ${ }^{25}$.

Genève sert aussi de tribune à l'écrivain, pour la publication de sa Lettre à Hauptmann ou de l'article "Au-dessus de la mêlée». Romain Rolland s'y installe donc le 3 octobre:

3 octobre 1914. Nous nous installons à Genève, Champel, Hôtel Beauséjour. Ma mère est avec moi; ma sœur et mon père, à une pension de Lancy. Mon intention, en venant ici, est de m'employer à une œuvre qui m’attire par son caractère humain, plus que national: l'Agence des Prisonniers de guerre, qui vient d'être fondée sous la direction de la Croix-Rouge internationale. Elle sert d'intermédiaires entre les prisonniers de toutes nations et leurs familles. Il y a quinze jours, j’ai écrit au président du CICR, Gustave Ador, pour lui demander de m'utiliser. Il m’a répondu (24 septembre), en me remerciant, "qu'il se ferait scrupule de recourir à mon concours, estimant que je rends par mes articles des services plus importants à la cause du libéralisme et au triomphe final des idées de justice et de progrès social». J'ai insisté et reçu ma convocation. Une heure après mon arrivée à Genève,

24. Romain Rolland apprend la nouvelle de la destruction de Louvain le 29. Cette nouvelle eut un grand retentissement, notamment en raison de l'incendie de la Bibliothèque qui avait une grande valeur artistique et intellectuelle.

25. $J A G$, p. 44. 
je vais à l'Agence. Elle est installée au palais Eynard, à l'extrémité de la promenade des Bastions. Ma première impression est un peu de désappointement. Trop de bureaucratie, avec cet esprit qui lui est propre, et qui semble s'appliquer à rendre tout ce que l'on fait plus difficile et plus lent ${ }^{26}$.

Romain Rolland travaille régulièrement au service de l'Agence des prisonniers de guerre jusque dans les premiers jours de juillet 1915. Comme l'a déjà montré Claire Basquin ${ }^{27}$, son départ est un faux départ, puisqu'il y reviendra travailler ensuite.

Rolland reversera par ailleurs à l'Agence un quart du montant de son Prix Nobel de littérature en juin 1917 - prix reçu pour l'année 1915.

Mais si Romain Rolland choisit Genève, c'est aussi pour y réunir les meilleurs esprits en une sorte de Parlement moral. Ce choix genevois est donc aussi pensé, voulu:

Je n'avais pas attendu la déclaration de guerre pour venir m'installer en Suisse. Mais j’ajoute hautement que si je n'y avais été déjà, j’y serais venu délibérément, afin de maintenir la clarté de ma vision et ma pleine liberté. Je n'accepte donc pas l'excuse de certains de mes amis qui disent que c'est le hasard qui m’a bloqué en Suisse. Ma volonté seule m'y a maintenu ${ }^{28}$.

Pour lui, le rôle d'un vrai Genevois est d'éclairer et de modérer la France. Il attend que Genève rapproche les mains qui se cherchent à travers l'espace. Il observe l'action de Gustave Ador et celle du Docteur Frédéric Ferrière, président et vice-président de la Croix-Rouge internationale, et croit sans doute que tout Genève suit l'ambition humaine de ces deux hommes. Cette croyance s'estompe au fur et à mesure que la guerre avance. Rolland note à propos du $\mathrm{D}^{\mathrm{r}}$ Ferrière, le 3 juillet 1915, «[...] le seul esprit de Genève (je le crois), qui soit resté juste, impartial et largement humain, au milieu du conflit universel ${ }^{29}$.

Malgré cette évolution de son opinion sur Genève, c'est bien de là, et plus généralement de Suisse, que Romain Rolland avait le sentiment de

26. Ibid., p. 73.

27. C. Basquin, Romain Rolland et l'Agence des prisonniers de Genève.

28. Cité dans S. Stelling-Michaud, "Le choix de Romain Rolland en 1914 », p. 26 sq.

29. $J A G$, p. 426. 
pouvoir mieux juger et participer à la guerre morale. C'est de Genève et de la Suisse qu'il pouvait accomplir son œuvre européenne.

Genève a permis à Romain Rolland de s'exprimer librement, la ville lui a fourni la tribune qu'il cherchait.

2. L'engagement de Romain Rolland et des réfugiés français à Genève pendant la guerre

\subsection{L'engagement de Romain Rolland en 1914 et 1915}

Le mois de juillet 1915 est une rupture dans le parcours de l'écrivain. Les moments difficiles traversés seul à Genève dans sa chambre d'hôtel le décident à quitter la ville pour retrouver le repos qu'il était venu chercher en Suisse. Les raisons sont multiples. Nous en avons déjà évoqué certaines. Il y a aussi sa croyance d'avoir échoué dans son appel:

7 juillet 1915. Voici le douzième mois que, placé au milieu de la guerre des nations, je m'efforce de défendre mon âme de l'injustice et d'en défendre les âmes qui sont dans le combat. Je n'oserais pas dire si j'ai réussi pour moi; mais je sais bien que je n’ai guère réussi pour les autres. Bien peu ont répondu à mon appel. [...] J'ai assez vu le monde des autres. J'ai le droit, à présent, de rentrer dans le mien ${ }^{30}$.

L'histoire du Journal de Genève qui refusa d'abord de lui réserver quelques colonnes pour son article Jaurès le décide aussi à quitter Genève. Son directeur (Wagnière) lui explique que le public ne comprendrait pas qu'un journal genevois accorde tant de place à cet évènement ${ }^{31}$. Romain Rolland y voit la politique du journal qui est celle, pour lui, de se mettre à la remorque des rancunes des nationalistes français.

Le 22 juillet 1915, Romain Rolland quitte Genève:

Je quitte Genève, où je vis depuis dix mois. Je suis dans un état de grande fatigue nerveuse. A l'angoisse des affaires publiques s'ajoutent des tourments personnels. Je vais chercher un peu de repos et de recueillement dans un coin de montagne, où je sois seul, à Château

30. $J A G$, p. 432.

3I. Il s'agit du premier anniversaire de la mort de Jean Jaurès. 
d'CEx. Toute la famille est dispersée: ma sœur à Schœnbrunn, mon père à Brunnen et ma mère à Genève ${ }^{32}$.

Ce départ change beaucoup de choses. A partir de celui-ci, Romain Rolland se sentira beaucoup moins atteint par les évènements. L'enseignement des douze premiers mois de la guerre est immense:

Depuis un an, j'ai sacrifié mon repos, mon succès, mes amitiés, à la tâche de combattre la déraison et la haine. J'ai essayé de faire sentir à chacun de nos deux ennemis - et spécialement aux miens - que ses adversaires sont des hommes qui souffrent comme lui. Chacun de mes articles m'a valu d'être outragé dans chacun des pays. Des deux côtés, je me suis heurté à la même incompréhension. Les outrages ne m'émeuvent pas, mais l'incompréhension à la fin, me désarme.

Je me retire momentanément d'une aveugle mêlée, où chacun des combattants n'écoute que sa propre passion et redit à tue-tête ses propres arguments, sans chercher le moyen de les rendre peu à peu accessibles aux autres [...] J'ai tenté l'impossible. Je ne m'en repens pas; c'était mon devoir de le tenter; mais je sens l'inutilité de persister, pour l'instant. Je me retire dans l'art qui reste le refuge inviolé; et j'attends que la folie du monde soit passée ${ }^{33}$.

L'action dans laquelle Romain Rolland s'était retrouvé entre août 1914 et juillet 1915 laisse place à un retrait dans le confort artistique que lui conférait sa position d'écrivain. Ce choix est définitif. Et finalement, la réussite lente et progressive de son appel n'y changera rien. Car au même moment, le front genevois laissé par Romain Rolland est repris par ses alliés et ses défenseurs spirituels, qui ont entendu les prises de position humanistes et internationalistes de Romain Rolland dans les journaux, particulièrement dans le Journal de Genève.

32. $J A G$, p. 446.

33. Cité dans La revue mensuelle, revue de Suisse romande dirigé par Charles Bernard, septembre 1915. 
2.2. La réussite d'un appel: l'arrivée de réfugiés français sur le front genevois à partir de 1915

Des réfugiés qui s'installent: juin-novembre 1915

Le premier de ce "groupe des réfugiés genevois " ${ }^{34}$ arrive le 3 juin 1915. Henri Guilbeaux vient à Genève pour travailler à l'Agence des prisonniers:

3 juin. Henri Guilbeaux arrive à Genève, où je lui ai trouvé, à l'Agence des Prisonniers, une place de secrétaire du Dr Ferrière. [...] il a obtenu non sans peine les papiers lui permettant de passer la frontière ${ }^{35}$.

Ensuite, Romain Rolland rencontre René Arcos le 13 août 1915. Né en 1881, il est écrivain et poète et fonde à la fin de la guerre les éditions du Sablier avec Frans Masereel ${ }^{36}$. René Arcos s'installera de temps à autre à Lausanne. Il se rend à Thoune pour y rencontrer Romain Rolland:

Visite de René Arcos. C'est un petit homme d'une trentaine d'années, replet, riant, très parisien d'aspect... Il partage mes idées. Mais il n’a pas la même façon de les sentir ${ }^{37}$.

Puis vint Gaston Thiesson, artiste peintre ${ }^{38}$. Ami de Romain Rolland avant la guerre, il fut à Paris son défenseur jusqu'en septembre 1915. C'est le mercredi 8 septembre 1915 que l'artiste arrive à Genève avant de s'installer ensuite à Vevey le mois suivant:

Gaston Thiesson arrive de Paris, le mercredi 8 septembre... Il m'apporte des nouvelles des quelques esprits français restés fidèles à l'idéal international ${ }^{39}$.

Charles Baudouin, Lorrain né en 1893, s'était rendu en Suisse en 1915 pour trois raisons: l'état de ses poumons, son désir d'enseigner dans

34. L'expression est utilisée par Romain Rolland lui-même dans l'introduction aux Poètes contre la guerre, p. 11.

35. JAG, p. 386.

36. Graveur et dessinateur belge.

37. $J A G$, p. 478.

38. Il signait certaines de ses lettres par la mention «Gaston Thiesson, écrivain et artiste peintre de Paris ".

39. JAG, p. 508. 
le nouvel institut pédagogique de Genève ${ }^{40}$, et son profond espoir d'y rencontrer Romain Rolland. Cette dernière raison est d'ailleurs peut-être la plus forte. En effet, ayant découvert l'appel de Rolland en août 1915 de Paris, Baudouin confie:

Elle disait (la voix de Rolland) tout haut ce que je pensais tout bas. [...] C'était un nouvel éclair, un nouveau signe du destin. Cela donna soudain une consistance bien plus forte à mon projet de Genève. [...] [L]e vœu de rencontrer Romain Rolland. Car il fallait le rencontrer ${ }^{41}$.

Cette rencontre se fait en octobre 1915. Le 11, Charles Baudouin s'était installé à la Chapelle, petit ensemble de villas et de fermes au-dessus de Carouge, dans le sud de Genève.

De même faut-il citer Claude Salives, ouvrier français et ancien mineur de Lens, qui se trouvait en Suisse depuis 1912. Il participe à l'action du groupe des réfugiés genevois, et écrit sous le pseudonyme de Claude Le Maguet.

Enfin, le poète Pierre-Jean Jouve s'installe en Suisse début novembre 1915. A Vevey dans un premier temps, où Romain Rolland lui rend visite:

2 novembre. De Genève, je vais à Vevey pour voir Jouve, qui vient d'arriver avec sa femme et son petit Olivier. Ils se sont installés pour quelques jours à la Tour de Peilz, en face de la maison de Thiesson; et ils doivent partir ensemble, dimanche, pour Montana ${ }^{42}$.

Un groupe de six personnalités venues s'installer à Genève ou à proximité en 1915 jouera donc un rôle dans le combat pacifiste lancé par Romain Rolland. Avec lui, ou parfois sans lui, ils sont «ses gardes du corps qui se lèvent, se dressent, croisent le fer et touchent les adversaires ${ }^{43}$.

40. Il s'agit de l'institut Jean-Jacques Rousseau, fondé en 1912 par le Genevois Edouard Claparède. Institut pour la recherche en psychologie et pédagogie et formation pour les éducateurs, cet institut devient Institut des Sciences de l'éducation en 1929 puis est incorporé à l'Université de Genève en 1948.

4I. Carnets de route, Genève, Bibliothèque publique et universitaire, fonds Charles Baudouin, ms. fr. 5963/2, p. 97.

42. $J A G$, p. 569.

43. Cité dans H. Guilbeaux, Pour Romain Rolland, p. 30. 
Nous en avons choisi six, du fait de leur action significative. D'autres étaient venus rencontrer Romain Rolland, mais n'avaient pas décidé de demeurer en Suisse.

Des réfugiés qui ne restent pas: 1915-1917

Il convient d'associer à ce groupe de réfugiés ceux qui, touchés par l'appel genevois de Romain Rolland, viennent en Suisse pour le rencontrer. De ces rencontres, une action nait et un combat se poursuit. Car en effet, des hommes comme Gustave Dupin, Fernand Desprès, ou Jean de Saint Prix, bien que restés en France, agiront via le groupe des réfugiés genevois venus en Suisse et particulièrement à Genève pour s'associer à Romain Rolland.

Le peintre et écrivain français Gustave Dupin par exemple rencontre Romain Rolland en mai 1915 après un échange de lettres de réflexion sur la guerre ${ }^{44}$. Son nom viendra souvent accompagner les actions du groupe des réfugiés de Genève.

Fernand Desprès se rend à Genève en mars 1916 pour passer huit jours et rencontrer Romain Rolland. Il déjeune avec lui et Henri Guilbeaux le 14. Fernand Desprès avait perdu sa place au journal La Bataille syndicaliste $^{45}$ car on lui interdisait d'y défendre Romain Rolland. Le compterendu de leur rencontre de mars 1916 est tout à fait intéressant:

Vu Fernand Desprès, mon défenseur à La Bataille syndicaliste où il perdit sa place de journaliste. Petit, jeune, assez trapu, la figure très large, tassée, jaune, et bouffie. On dirait un paysan du centre de la France, ou un prêtre. Il est venu pour une huitaine, surtout afin de m'apporter le volume de Marcelle Capy: Une voix de femme dans la

44. Gustave Dupin écrit pour la première fois à Romain Rolland le 22 décembre 1914, ayant perdu son fils au front: "Je vous prie d'accueillir les compliments d'un obscur qui pense comme vous... J'ai eu la douleur de perdre mon cher fils dans cette tourmente effroyable. [...] Nous aurions trouvé plus de repos et moins d'amertume même chez les sauvages, que dans cette Europe prétendue civilisée! Mais ce n'est pas cette douleur qui m’a rendu pacifiste. Je l'ai toujours été», JAG, p. 195.

45. La Bataille syndicaliste est un journal du mouvement ouvrier français qui à partir de 1915 mettra en place une censure officieuse exercée par certains syndicalistes du journal. 
mêlée ${ }^{46}$. J'en dois écrire la préface; mais les épreuves qui m’ont été envoyées par la librairie Ollendorff n’ont jamais pu me parvenir: la censure les intercepte ${ }^{47}$.

Nous reviendrons plus loin sur l'attachante figure de Jean de Saint-Prix.

Romain Rolland fut donc soutenu au moment où il s'y attendait le moins. Ce groupe de réfugiés venant combattre à ses côtés fut cette poche de résistance en dehors de la France. Mais si Romain Rolland s'était fixé comme règle de "ne jamais faire partie d'un groupe " ${ }^{48}$, chose qu'il rappellera au cours de la guerre, il semble bien qu'il ait aussi appartenu au groupe des francs-tireurs français et ait soutenu leur combat pacifiste.

Le «nous» qu'il utilise témoigne de sa solidarisation et identification à ce groupe des francs-tireurs français. La volonté de Romain Rolland de ne pas s'associer au mouvement ou groupe se réclamant de lui n'empêcha pas la constitution d'un groupe de dissidents français en Suisse à partir de 1915.

\subsection{Un groupe de dissidents français}

De jeunes hommes en quête d'un idéal

La première caractéristique de ce groupe est qu'il est essentiellement constitué d'hommes. Une autre caractéristique est l'âge de ces hommes. On est surpris par leur jeunesse. Romain Rolland fait alors figure de "père spirituel» (il a 49 ans en 1915):

Et Rolland deviendra pour Baudouin - qui avait perdu son propre père à l'âge de onze ans - un de ses "pères vivants» chez qui il cherchera «l'appui nécessaire» ${ }^{49}$.

46. Marcelle Capy était aussi journaliste à La Bataille syndicaliste. Elle démissionna comme Fernand Desprès du journal en août 1915, car la censure syndicaliste trouvait "qu'on parle trop de Romain Rolland, cela n'intéresse personne», JAG, p. 526.

47. Ibid., p. 710.

48. R. Rolland, Mémoires et fragments du journal, p. 277.

49. Correspondance entre Romain Rolland et Charles Baudouin, introduction d'Antoinette Blum, p. 14. 
Baudouin n'est pas seul parmi cette jeune génération à rechercher un idéal pacifiste et humaniste. Ainsi Romain Rolland semble pleinement jouer son rôle de maître des futures générations au cours de cette guerre.

Car la génération que son appel fait venir à Genève n'est pas la sienne: Jean de Saint-Prix a 21 ans, Charles Baudouin 22. Claude Salives et Pierre-Jean Jouve ont tous deux 28 ans, Henri Guilbeaux 31 et René Arcos 34. Seul Gustave Dupin est à peu près du même âge. Ce groupe relativement jeune est prêt à agir aux côtés de Romain Rolland, et surtout prêt à le suivre dans son combat pacifiste lancé à Genève:

A Genève, cependant, un petit groupe de jeunes écrivains se forma autour de lui ; ils étaient ses élèves et devinrent ses amis, puisant de la force dans la sienne. Le premier d'entre eux, Pierre-Jean Jouve, René Arcos, qui comme lui, avait vu la guerre dans son horreur et la détestait tout autant; Charles Baudouin, lui, exalte l'éternelle bonté; le graveur belge Frans Masereel taille sur ses planches la plainte de l'humanité, imagier grandiose de son temps, plus humain dans ses protestations dessinées que tous les livres et tous les tableaux; Guilbeaux, fanatique de l'essor social, véritable coq de combat dressé contre toute espèce de puissance, fonde une revue mensuelle, Demain, la seule vraiment européenne, avant qu'elle ne sombre tout à fait dans la pensée russe; Claude Le Maguet fonde les Tablettes qui, grâce à de hardis collaborateurs et aux dessins de Masereel, deviennent le plus vivant des périodiques paraissant alors en Suisse ${ }^{50}$.

Ce long hommage de Stefan Zweig montre le caractère jeune de ce groupe et son envie de combattre auprès de Romain Rolland. Il montre aussi son caractère littéraire: la plupart sont écrivains, artistes, ou peintre, et agiront par la voie journalistique. S’ils décident de venir à Genève, c'est aussi pour pouvoir parler et s'exprimer.

Des réformés ou démobilisés, écrivains ou artistes à la santé fragile

Les raisons qui poussèrent les acteurs de ce groupe de réfugiés français à venir s'installer en Suisse sont d'ordres différents. Une santé fragile par exemple pour Pierre-Jean Jouve qui fut très lié à Romain Rolland et avec qui il partageait les convictions pacifistes internationalistes.

5o. S. Zweig, Romain Rolland, p. 311. 
René Arcos lui, était correspondant du journal Chicago Daily News pendant la guerre et venait de temps en temps rendre visite à Romain Rolland.

Henri Guilbeaux, né en 1884, auteur d'œuvres sur la littérature allemande et journaliste, prend position en 1914 pour le pacifisme et l'internationalisme ${ }^{51}$. Il fut un des premiers défenseurs de Romain Rolland pendant la guerre avec l'article «Lettre ouverte à Romain Rolland" publié dans La Bataille syndicaliste. Il créa la revue Demain, foyer d'opposition à la guerre.

Enfin, il est permis de citer de nouveau Jean de Saint-Prix, jeune écrivain et journaliste français réformé, conduit par l'idéal pacifiste et intellectuel montré par Romain Rolland.

Né le 25 septembre 1896, Jean de Saint-Prix était le petit-fils d'Emile Loubet ${ }^{52}$. Reçu bachelier latin-grec-philosophie, il s'inscrit à la Sorbonne en novembre 1914; huit mois plus tard, il passe la licence ès lettres (philosophie) avec la mention «Bien». Exempté deux fois du service militaire en raison de sa santé très fragile, désespéré par la guerre, il vit en 1915 et 1916 deux années de véritable agonie morale. Ayant obtenu non sans peine d'un fonctionnaire du Quai d'Orsay qu'il connaissait un passeport pour la Suisse, il y arrive le 29 juillet 1917 et y passe trois semaines, principalement à Genève. C'est en août à Villeneuve qu'il rencontre pour la première fois Romain Rolland, lequel est un peu méfiant au début:

Sa généreuse et naïve ardeur me fait plaisir et me peine, car elle me rappelle mes propres illusions et mes souffrances, à son âge; et je prévois pour lui bien des épreuves, que je voudrais lui épargner. Comme je lui ai conseillé de ne pas se compromettre inutilement parmi nous, puisqu'il doit retourner en France, il me répond de Genève ... ${ }^{53}$

Mais les premières préventions sont vite dépassées:

Cher Jean de Saint-Prix

C'est parce que j'ai reconnu en vous un frère plus jeune, que je voudrais vous épargner quelques-unes de mes épreuves. Mais si je ne le

5I. L'internationalisme est une doctrine philosophique et politique qui s'oppose au nationalisme et prêche soit la coopération entre tous les Etats et tous les peuples du monde, soit la suppression des frontières.

52. Emile Loubet fut Président de la République de 1899 à 1906.

53. JAG, p. 1287. 
puis, mon affection vous y accompagnera. Vous êtes un généreux garçon. Je vois au fond de vous bien des larmes cachées. Mais leur source est faite d'amour et de douleur. Et mieux vaut l'un et l'autre qu'être privé des deux. Qu'elle ne tarisse donc jamais.

Vous êtes moins seul en France que vous ne croyez ${ }^{54}$.

Leur correspondance se poursuivra tout au long de la guerre. On y apprend les actions éditoriales des uns et des autres, le fait que Romain Rolland s'applique à recopier certains manuscrits de jeunes francs-tireurs français comme celui de Marcel Martinet en août 1917 ou à corriger des épreuves de Gustave Dupin.

Jean de Saint-Prix sera emporté par la grippe espagnole et mourra le 18 février 1919.

\section{Un groupe homogène ou désuni?}

La présentation succincte du groupe des réfugiés français a permis de mettre en lumière ses grandes caractéristiques. Il est jeune, à l'esprit artistique et intellectuel, réformé ou démobilisé et par conséquent assez loin d'avoir connu la difficulté et les conditions de vie au front.

Ce groupe est donc inexpérimenté, prêt à agir suivant les aspirations du cœur. Ils seront ces "dissidents» français qui tenteront de rappeler à l'Europe, ou tout du moins à la France, la vérité et l'humanité. Tentative difficile aussi du fait de leur position géographique en Suisse qui les empêchera d'avoir une grande influence. Tentative difficile du fait que la plupart d'entre eux sont des inconnus et des anonymes même parfois: qui connait en 1915 Charles Baudouin, Henri Guilbeaux, ou Gaston Thiesson?

René Arcos et Pierre-Jean Jouve semblent un peu plus connus dans le milieu des écrivains parisiens, mais soulignons que leur relative notoriété après-guerre sera plus due à leur production littéraire qu'à leur combat pacifiste pendant la Première Guerre mondiale.

Cependant, ce groupe exista bel et bien. Et s'il exista, c'est sans nul doute grâce à Romain Rolland qui fit figure de rassembleur des

54. Cité dans En plein vol, Jean de Saint-Prix et Romain Rolland, textes et documents, p. 20. 
pacifistes. Sans le vouloir, il attira à lui de jeunes hommes qui allaient combattre intellectuellement avec lui.

\section{L'aventure ordinaire d'un intellectuel franc-tireur, autorité morale malgré lui}

3.1. Les précautions prises par Romain Rolland à l'égard de ses alliés

J'ai eu à me défendre contre mes alliés, pendant la guerre, beaucoup plus que contre mes ennemis, et c'est je crois une aventure ordinaire ${ }^{55}$.

Cette révélation de Romain Rolland au cours de la guerre est symbolique de sa volonté d'être seul et d'agir seul. Car cette "aventure ordinaire» fut une résistance constante à toute appropriation ou utilisation de son nom ou de ses paroles par un groupe ou mouvement pacifiste qu'il avait luimême contribué à former.

Par exemple, dans sa relation au Carmel, Romain Rolland a dû prendre tout de suite des précautions avec Charles Baudouin, son directeur:

D'autre part, Guilbeaux m'a dit que vous lui aviez parlé de ma collaboration à votre revue. Je ne suis pas collaborateur à votre revue, pas plus qu'à celle de Guilbeaux, ou à toute autre revue. [...] Je n'ai jamais fait et ne ferai jamais partie d'aucun groupe, artistique ou politique. Je suis, par essence, indépendant irréductible, et tiens à le rester d'autant plus, en une époque où sévit l'associationnisme, avec toutes les conséquences morbides d'une mentalité collective ${ }^{56}$.

Baudouin tenta donc en vain de s'assurer la collaboration active de Rolland, ce dernier lui opposant un refus catégorique. Cette attitude de Romain Rolland sera caractéristique jusqu'au début des années 1930, Rolland craignant en effet toute association officielle limitant sa liberté d'esprit.

55. Cité dans Ph. Monnier, «Romain Rolland et la Revue mensuelle de Genève», p. 5.

56. Lettre de Romain Rolland à Charles Baudouin, 10 février 1916, in A. Blum (éd.), Correspondance entre Romain Rolland et Charles Baudouin, p. 36. 
Henri Guilbeaux en fera aussi les frais. Dès le début de Demain, la revue se place sous l'égide de Romain Rolland et celui-ci en est agacé. Car dans la partie documentaire de Demain, le nom de Rolland revient très souvent. Une note sur son anniversaire apparaît, ce qui ne plaît guère à l'écrivain.

Il analyse cette note comme de la publicité indiscrète et devra écrire à Guilbeaux pour que cessent ces mentions trop fréquentes du nom de Romain Rolland:

Mon rôle est tout à fait secondaire; et, en tout cas, il est passé. Il n’y a pas lieu d'y revenir sans cesse. Quand dix millions d'hommes s'égorgent, il est déplacé de parler autant d'un plumitif. J'ai le droit de le traiter ainsi, puisque c'est moi; et ce n'est rien de plus. Il a fait son devoir de conscience en septembre 1914. Un point, c'est tout. Passons à autre chose. A l'avenir, ne parlez donc plus de lui, à moins qu'il ne s'agisse de réfuter une calomnie précise et pressante ${ }^{57}$.

Malgré les précautions, les attaques iront bon train et l'association sera rapidement faite entre l'internationalisme de Guilbeaux et l'écrivain Romain Rolland.

Des précautions qui sont aussi prises à l'égard de La Revue mensuelle de Charles Bernard. Cette revue avait publié entre janvier 1916 et mars 1917 une grande enquête sur l'attitude de Romain Rolland pour prouver que celui-ci n'était pas isolé et que ses vœux, angoisses, et espoirs étaient partagés. Cette enquête inédite et longue avait été dirigée par Romain Rolland lui-même. Mais une fois de plus, l'enquête était allée trop loin, avait trop parlé de lui, avait cité son nom trop souvent.

Dès le 11 août 1916, Rolland alerte Bernard: «Il me semble qu'on a déjà trop longtemps parlé de moi. Il ne faut pas abuser de la patience du public. ${ }^{58}$.

Car, en effet, de nombreuses lettres de soutien à Romain Rolland étaient parvenues à La Revue mensuelle et avaient été présentées comme des documents historiques. Bientôt emporté par son enthousiasme,

57. JAG, p. 663.

58. Cité dans Ph. Monnier, "Romain Rolland et La Revue mensuelle de Genève", p. 4 sq. 
Charles Bernard sera amené à les publier et à dire des choses sur Romain Rolland qui ne seront pas fondées ${ }^{59}$.

La précaution se transforme ainsi en défense et résistance de la part de Rolland à l'égard de ses alliés et amis. Bernard reprochait par exemple à Rolland de lui préférer d'autres revues comme Le Carmel. Il lui reprocha aussi la publication de l'article "Aux peuples assassinés" dans la revue Demain. Romain Rolland a dû donc aussi démontrer à ses alliés qu'ils n'étaient pas rivaux les uns des autres: "Les idées sont entièrement différentes. Demain est franchement, violemment révolutionnaire.» ${ }^{60}$.

Et voici que Romain Rolland s'érige en conciliateur entre ses différents camarades. Leurs actions individuelles entraînèrent en effet parfois des désaccords internes au sein du groupe des réfugiés français. En particulier entre Guilbeaux et Jouve:

Je passe des jours à empêcher ces compagnons de lutte de s'entredéchirer. Guilbeaux est bien intolérant, mais Jouve est bien inconséquent; et tous deux aussi susceptibles et aussi hypersensibles, ont de constants malentendus, qui frisent la guerre déclarée... Tous d'eux m'écrivent, chaque jour, m'exposant chacun les choses avec son tempérament propre: Guilbeaux, implacable, mais droit et ferme; Jouve, gémissant et vibrant, comme des roseaux sous le vent ${ }^{61}$.

Des querelles éditorialistes aux méfiances des uns vis-à-vis des autres, Romain Rolland est au centre, arbitre des divisions et incompréhensions des membres du groupe des réfugiés français que lui-même avait plus ou moins contribué à former. Cette résistance ne fut pas prévue au départ par Rolland. Il savait que son appel et ses paroles de 1914 susciteraient des critiques et attaques, mais ignorait sans doute qu'il aurait dû, aussi, se défendre de ses alliés.

De même, un projet de "Société des amis de Romain Rolland" fut avancé, ce qui lui déplut immédiatement. Pierre-Jean Jouve lui rappelle ce projet:

59. "Vous racontez que... C'est absolument faux... Comment pouvez-vous annoncer un fait qui n'a rien de fondé? ", lui écrit Rolland (ibid., p. 8).

6o. Ibid., juin 1917, p. 8.

6. JAG, p. 1406. 
C'est encore moi. Je vous écris pour vous transmettre une idée de Dupin sur sa demande.

Vous savez qu'il avait pensé, il y a deux ans, à fonder «Société des amis de Romain Rolland", et qu'il avait renoncé à cette idée sur le conseil de votre mère et de votre sœur. Or il y repense en ce moment, et cela lui tient à cour. Il me prie de vous exposer la manière dont il conçoit la chose $[\ldots]^{62}$

On devine aisément la réponse que Romain Rolland fit en rapport à ce projet:

Cher Jean de Saint-Prix

Merci de vos affectueuses lettres. Vous aurez su par G.D. et par M.M. que je ne serai jamais des Amis de R.R. Vous vous en doutiez bien, je suppose! Non, vous n'aurez jamais à craindre cela de moi. Pas plus que de me voir quémander à la porte d'une Académie. J'aime trop ma liberté, et je n’arrive jamais à en avoir assez. Pas de parc à moutons! ${ }^{63}$

Le combat que Romain Rolland dut mener contre ses propres alliés, combat incessant à l'égard d'articles, de pensées, de prises de position de ses amis qui tentaient de poursuivre la bataille morale lancée par ce dernier, fut donc une épreuve redoutable à laquelle Romain Rolland ne s'attendait pas. Contraint de se défendre afin de préserver l'authenticité et la nature même de ses propres prises de position, nous comprenons aisément la difficulté pour ses alliés et amis de porter son message: Romain Rolland ne souhaitait pas qu'on le porte, il souhaitait le porter lui.

\subsection{Romain Rolland, incarnation d'une autorité morale malgré lui}

Poursuivant son activité d'écrivain, prolongeant son séjour en Suisse au moment où la guerre éclate, Romain Rolland devient rapidement le symbole d'un combat contre la haine des nations et pour la défense de valeurs humaines menacées. Son message prend rapidement une telle ampleur et acquiert un tel rayonnement qu'il se retrouve prisonnier de son image en Suisse. Ce message devient tout à la fois: message pacifiste, humain, moral, politique, destiné à l'ensemble des acteurs en présence.

62. En plein vol, Jean de Saint-Prix et Romain Rolland, textes et documents, p. 47.

63. Id., p. 51 sq. Il s'agit probablement de Gustave Dupin et de Marcel Martinet. 
Romain Rolland est d'abord écouté, puis entendu et rejoint par d'autres acteurs qui souhaitent poursuivre avec lui la tentative intellectuelle de grouper des voix dissidentes à travers l'Europe. Il apparaît alors comme le symbole et le chef désigné de cette volonté partagée, rôle qu'il refuse d'endosser comme nous avons pu le voir.

Il incarne une autorité morale qui le dépasse:

1. Nous pouvons, aujourd'hui, affirmer que Romain Rolland n'est pas un «isolé» et que ses paroles, au lieu d'être déprimantes, sont allés au cœur des hommes et ont allégé leurs misères. Gaston Thiesson

2. C'est pourquoi la noble attitude de Romain Rolland est non seulement réconfortante à un moment où il n'y a pas seulement de la noblesse et de l'héroïsme dans les spectacles qui nous entourent, mais encore significative de la seule paix qui vaille le poids d'or de la réalité perdue, et que nous retrouverons si nous nous acheminons vers elle avec la largeur d'âme et d'esprit qui convient. Soldat L.B

3. Je pense que parmi les intellectuels de chez nous qui écrivent pendant cette guerre et sur cette guerre, Romain Rolland apparait comme l'un des mieux français et dignes de la France. Caporal Ch.V.

4. Je salue un frère d'armes dans l'homme qui a tendu ses forces vers ce but: la défaite de l'esprit de jalousie, de méconnaissance, d'injure, de mépris et de haine réciproque entre les deux peuples, la défaite de l'esprit d'entreprise matérielle contre les libertés voisines. Sous-lieutenant J.-R. Bloch ${ }^{64}$.

Ces nombreux témoignages prouvent à la fois la portée de sa pensée et la très grande résonnance que Romain Rolland eut auprès de nombreux soldats du front. Il revenait alors à l'intellectuel français de se montrer à la hauteur des attentes de tous ceux qui partageaient avec lui le fond de sa pensée. Il devait jouer un rôle qu'il n'avait pas demandé, pas souhaité, mais que les observateurs lui demandaient d'incarner. Cette ambiguïté fut donc à l'origine de cette aventure ordinaire. Rolland ne souhaitait pourtant pas l'endosser.

Il ne souhaitait ni être enrôlé, ni être le représentant moral du rapprochement des pensées libres d'Europe. La dichotomie perpétuelle entre l'état d'écrivain, de penseur, de moralisateur et celui d'intellectuel engagé se manifeste chez Romain Rolland dès le début de la guerre, alors que son message trouve un écho favorable. Conscient de cet écho, il

64. JAG, p. 539-546. 
utilise alors la tribune qui lui est offerte pour asséner un discours humain et moral, moralisateur presque. Il parvient ainsi à transmettre par la même occasion un discours profondément politique accusant à la fois les gouvernements en place, les intellectuels et l'Eglise qui ne réagissent pas face au déclenchement du conflit.

Romain Rolland vise ainsi les intellectuels français qui prennent parti pour la guerre en entretenant la haine de l'Allemagne. Tout en dramatisant la trahison des intellectuels, il souhaite incarner le rôle de porte-voix d'un idéal de justice que d'autres intellectuels avaient abandonné.

Malgré lui donc, il «s'autoattribue» une autorité morale qui est à la fois une réalité étant donné la portée de son message, mais aussi une sacralisation du rôle qu'il croit devoir symboliser.

Il est intéressant de souligner que l'analyse historique de Romain Rolland se fait dès novembre 1914, analyse qui rejette la responsabilité sur la France et l'Allemagne. Elle rejoint ainsi celle qui plus tard sera proposée par le courant historiographique représenté par Pierre Renouvin. La publication dans la Revue d'histoire de la Guerre mondiale entre 1923 et 1939 du vaste travail aboutissant à la thèse d'une responsabilité partagée fait écho aux idées sous-entendues par Romain Rolland dès novembre 1914.

L'intellectuel français poursuit son travail introspectif. Il se veut exemplaire dans ses prises de position, et reproche à ses pairs de ne pas le suivre. Il accuse directement les intellectuels :

Leur rôle dans cette guerre a été affreux; on ne saurait leur pardonner. Non seulement ils n'ont rien fait pour diminuer l'incompréhension mutuelle, pour limiter la haine; mais à bien peu d'exceptions près, ils ont tout fait pour l'étendre et l'envenimer. Cette guerre a été, pour une part, leur guerre. Ils ont empoisonné de leurs idéologies meurtrières des milliers de cerveaux. Sûrs de leur vérité, orgueilleux, implacables, ils ont sacrifié au triomphe des fantômes de leur esprit des millions de jeunes vies. L'histoire ne l'oubliera pas ${ }^{65}$.

Il aura par ailleurs cette formule très dure à l'encontre des clercs qu'il compare à un troupeau totalement soumis et aveugle:

65. Romain Rolland, Les Précurseurs, p. 197. 
Je ne demandais qu'une voix, une seule, qui fût libre... Aucune voix n’a parlé. Et je n'ai entendu que la clameur des troupeaux, les meules d'intellectuels aboyant sur la piste ou le chasseur les lance [...] Que vous êtes maladroits! Je crois que de tous vos défauts, la maladresse est la pire. Vous n'avez pas dit un mot, depuis le commencement de cette guerre, qui n'ait été plus funeste pour vous que toutes les paroles de vos adversaires. Les pires accusations qu'on ait portées contre vous, c'est vous qui en avez fourni, de gaieté de cour, la preuve ou l'argument ${ }^{66}$.

L'attaque est acerbe de la part de Romain Rolland. Son ressentiment à l'égard des intellectuels et des penseurs est à la mesure de sa conception du rôle de l'intellectuel engagé qui doit éclairer les autres par ses prises de position et sa capacité à véhiculer un idéal de justice.

L'autorité morale que Romain Rolland incarne depuis la Suisse est donc délicate à cerner, s'agissant de son rayonnement en France. Ses prises de position très fermes à l'encontre de ses compatriotes intellectuels ont forcément engendré une haine à son encontre. Sa posture d'opposant, sorte de conscience de l'Europe, qui s'évertue à demeurer au-delà des clans des nations, ne pouvait que générer une profonde antipathie en France, où régnait alors un sentiment de nationalisme exacerbé, y compris bien sûr chez les intellectuels.

Pour autant, cette conscience de l'Europe allait bel et bien trouver un écho dans de nombreux pays. Au-delà des frontières suisses, l'intellectuel français allait toucher de très nombreux esprits qui bientôt décidèrent de se mettre en contact avec lui, de lui écrire, de lui rendre visite, ou de relayer son message: un commerce spirituel international se mettait alors en place suite à l'appel solitaire de Romain Rolland qui entendait par là créer un vaste mouvement collectif. Cet appel, quant à lui, ne fut possible que précisément dans le contexte suisse de la Grande Guerre.

Guillaume Juin

Université Paris IV - Sorbonne

66. Romain Rolland, Au-dessus de la mêlée, p. 38. 


\section{BIBLIOGRAPHIE}

\section{Sources}

Blum, Antoinette (éd.), Correspondance entre Romain Rolland et Charles Baudouin: une si fidèle amitié: choix de lettres (1916-1944), avantpropos d'Yves Baudouin, Meyzieu, Césura, 2000.

Chère Sofia: choix de lettres de Romain Rolland à Sofia Bertolini GuerrieriGonzaga (1909-1932), t. II, texte établi par Marie RomainRolland, préface d'Umberto Zanotti-Bianco, Albin Michel, 1960 (Cahiers Romain Rolland 11).

Correspondance entre Louis Gillet et Romain Rolland, choix de lettres établi par $\mathrm{M}^{\mathrm{me}}$ Louis Gillet et $\mathrm{M}^{\mathrm{me}}$ Romain-Rolland, préface de Paul Claudel, Paris, Albin Michel, 1949 (Cahiers Romain Rolland 2).

En plein vol, Jean de Saint-Prix et Romain Rolland, textes et documents, texte établi par Marie Romain-Rolland et par Pierre de Saint-Prix, préface de Pierre de Saint-Prix, Paris, Albin Michel, 1980 (Cahiers Romain Rolland 25).

Fonds Charles Baudouin, Bibliothèque publique et universitaire de Genève.

Guilbeaux, Henri, Pour Romain Rolland, Genève, J.-H. Jeheber Librairie Editeur, 1915.

Rolland, Romain, Les Poètes contre la guerre, Genève, Editions du Sablier, 1920.

—, Les Précurseurs, Paris, Editions de l'Humanité, 1920.

—, Au-dessus de la mêlée, Paris, Albin Michel, 1926.

—, Journal des années de guerre, Paris, Albin Michel, 1952.

—, Mémoires et fragments du journal, Paris, Albin Michel, 1956.

- Je commence à devenir dangereux: choix de lettres de Romain Rolland à sa mère (1914-1916), introduction d'Else Hartoch, Paris, Albin Michel, 1971 (Cahiers Romain Rolland 20). 


\section{Travaux}

Audouin-Rouzeau, Stéphane, Becker, Jean-Jacques (éds), Encyclopédie de la Grande Guerre 1914-1918, Paris, Bayard, 2004.

BAsquin, Claire, Romain Rolland et l'Agence des prisonniers de Genève, Thèse, Paris, Ecole Nationale des Chartes, 1999.

Fleury, Antoine, "Genève: lieu de mémoire d'une Europe rêvée et de l'Europe des réalités", in Penser l'Europe, 40 ans d'études européennes à Genève, Genève, Institut européen de l'Université de Genève, 2003, p. 89-96.

Histoire de Genève de 1798 à 1931, Genève, Imprimerie du Journal de Genève, 1956.

Monnier, Philippe, «Romain Rolland et la Revue mensuelle de Genève», Etudes de Lettres, 3 (1976), p. 1-13.

Romain Rolland et la Suisse, Catalogue d'exposition, Musée d'art et d'histoire, Genève, 1966.

Stelling-Michaud, Sven, "Le choix de Romain Rolland en 1914 ", La Pensée. Revue du Rationalisme Moderne, 132 (avril 1967), p. 23-30.

Zweig, Stefan, Romain Rolland, Paris, Belfond, 2000. 
